\title{
A Subject-Specificity Analysis of Radio Channels in Wireless Body Area Networks
}

\author{
Yan $\mathrm{Zhao}^{1, *}$ and Yang $\mathrm{Hao}^{2}$ \\ 1 International School of Engineering, Faculty of Engineering, Chulalongkorn University, \\ 254 Phayathai Road., Pathumwan, Bangkok 10330, Thailand \\ 2 School of Electronic Engineering and Computer Science, Queen Mary University of London, \\ Mile End Road, London E1 4NS, United Kingdom \\ E-mail: yan.z@chula.ac.th*
}

\begin{abstract}
This paper presents an analysis of subject-specific radio channels in wireless body area networks (WBANs) using a simulation tool based on the parallel finite-difference time-domain (FDTD) technique. This technique is well suited to model radio propagations around complex, inhomogeneous objects such as the human body. The impacts of different subjects varying in size on on-body, inter-body, and offbody radio channels are studied. The analysis demonstrates that the characteristics of on-body radio channels are subject-specific and are associated with human gender, height, and body mass index. On the other hand, when waves propagate away from the body, such as in the inter-body and off-body cases, the impacts of different subjects on the channel characteristics are found to be negligible.
\end{abstract}

Keywords: Body area networks, finite-difference time-domain method, radio propagation, subject-specific.

ENGINEERING JOURNAL Volume 15 Issue 3

Received 25 March 2011

Accepted 20 May 2011

Published 1 July 2011

Online at http://www.ej.eng.chula.ac.th/eng/

DOI:10.4186/ej.2011.15.3.39 


\section{Introduction}

Over the past decade, advances in wireless sensor technologies propelled by micro-electro-mechanical systems (MEMS)-based miniaturization and cost reduction have made pervasive wireless sensor networks (WSNs) a reality. Integrated microsensors of no more than a few millimeters in size, with onboard processing and wireless data transfer capability are the basic components of such networks.

The ultimate aim of wireless body area networks (WBANs) is to provide a truly personalized monitoring platform that is pervasive, intelligent, context-aware, and 'invisible' to the patient, thereby avoiding activity restriction or behavior modification. It is expected that it will attract a range of applications, from monitoring of patients with chronic disease and care for the elderly, to general wellbeing monitoring and performance evaluation in sports [1-5]. Before its widespread use, however, there are a number of technical challenges that need to be tackled. These include the need for better sensor design, MEMS integration, biocompatibility, power source miniaturization, low power wireless transmission, context awareness, secure data transfer, and integration with therapeutic systems.

While wireless communications through the air have been extensively documented, the communication from mounted/implanted devices around the human body is a new area of study. To ensure an efficient performance of WBANs, the propagation channels need to be characterized and modeled. Many efforts have been made to experimentally characterize the on-body radio channel for narrowband [6-9] and ultra-wideband (UWB) systems [10-12]. However, measurements are expensive, time consuming, and hardly repeatable. Computational models were developed to provide a physical insight of the on/in-body radio channel mainly by using the finite-differences time-domain (FDTD) technique [13]. In [14, 15], the FDTD technique has been used to analyze the radiation characteristics of implanted devices. The FDTD modeling of body-worn systems has been presented in [16-19]. In [17, 18] the body-worn performances of planar antennas have been analyzed. In [20], CST Microwave Studio $^{\mathrm{TM}}$, which is based on the finite integral technique (FIT), has been used to analyze the bodymounted performances of a compact sensor deployed in health-care applications.

In this paper, a simulation environment based on the parallel FDTD method is presented. The developed model is able to handle complex and electrically large problems, as well as take into account the radiation characteristics of the antenna in radio channels. It provides an efficient solution to radio channel modeling for WBANs, which is not available using commercial simulation packages without requesting excessive computational resources. This paper also aims to investigate the impact of different digital body models on radio channels in WBANs. Three different scenarios have been considered, namely, the on-body, inter-body, and off-body radio channels.

\section{Description of the Simulation Model}

The FDTD method is one of the most popular electromagnetic numerical techniques, and is well suited for modeling complex and irregularly shaped objects such as the human body. The FDTD is a versatile technique, but similar to other numerical methods, it is computationally intensive. For large EM problems such as the modeling of WBANs, the requirement for system resources is beyond the capability of personal computers (PCs). One way to resolve this problem is to divide the whole computational domain into smaller sub-domains, and each sub-domain can be handled by a single PC. By linking the PCs together with a proper synchronization procedure, the original large problem can be decomposed and solved efficiently.

One of the most attractive features of the FDTD method is that it can be easily parallelized with very little modification to the algorithm. Since it solves Maxwell equations in the time-space domain, the parallel FDTD algorithm is based on the space decomposition technique. The data transfer functionality between processors (PCs) is provided by the message passing interface (MPI) library. Data exchange is required only for the adjacent cells at the interface between different sub-domains and is performed at each time step, hence the parallel FDTD algorithm is a self-synchronized process. At the end of parallel FDTD simulations, the results calculated at each node are combined to obtain the final simulation result. The GCC compiler and a free version of MPI, MPICH, developed by Argonne National Laboratory [21], are used to compile our parallel FDTD code and handle the inter-node communications, respectively. 
In FDTD simulations, regular objects such as spheres, cylinders and cubes can be mathematically defined in space and easily represented in the FDTD domain. However complex objects such as the human body are often difficult to model and sometimes approximated by basic geometries [22]. While for realistic simulations, proper human body models need to be created and imported to the FDTD domain. In literature, several solutions have been proposed to computationally model the human body. In $[23,24]$ a flat uniform phantom representing the muscle tissue has been applied. In [19] a realistic uniform body phantom called James is used. A stratified dielectric phantom - HUGO model based on the data provided by the Visible Human Project of the National Library of Medicine [25] has been used in [20]. In [16], simulations are performed considering a male and a female Japanese inhomogeneous body models developed from the National Institute of Information and Communication Technology (NICT) [26].

The use of a uniform dielectric phantom rather than a stratified one, is justified from the fact that at $2.4 \mathrm{GHz}$ the human body is very lossy and the skin depth is very small, so the internal composition of the body is not very important. This way, largely available human body models as well as other objects from popular computer aided design (CAD) software can be readily imported to use in FDTD simulations. In total nine subjects have been considered in our analysis: five female and four male models with different dimensions, as listed in Table 1.

Table 1. The dimensions of nine subjects scanned using MRI and used in FDTD simulations ( $\mathrm{F}-$ Female, M-Male).

\begin{tabular}{c|ccccccccc}
\hline & F01 & F02 & F03 & F04 & F05 & M01 & M02 & M03 & M04 \\
\hline Height $(\mathrm{m})$ & 1.60 & 1.66 & 1.55 & 1.65 & 1.80 & 1.76 & 1.67 & 1.78 & 1.80 \\
Weight $(\mathrm{kg})$ & 50 & 55 & 52 & 52 & 75 & 73 & 56 & 87 & 85 \\
BMI & 19.5 & 20.0 & 21.6 & 19.1 & 23.1 & 23.6 & 20.1 & 27.5 & 26.2 \\
Waist $(\mathrm{cm})$ & 67.0 & 72.7 & 68.8 & 66.2 & 81.9 & 82.6 & 67.1 & 91.0 & 84.2 \\
Chest $(\mathrm{cm})$ & 79.4 & 85.6 & 96.9 & 80.7 & 107.9 & 91.3 & 82.1 & 101.1 & 98.4 \\
\hline
\end{tabular}

The subjects are scanned using magnetic resonance imaging (MRI) and a surface mesh is generated for each subject. However, since volume meshes are required in FDTD simulations, a voxelizer tool [27] has been used to convert surface meshes into volume ones. Figure 1 shows the cross sections of the nine voxelized subjects (at the height of the subjects' stomachs).

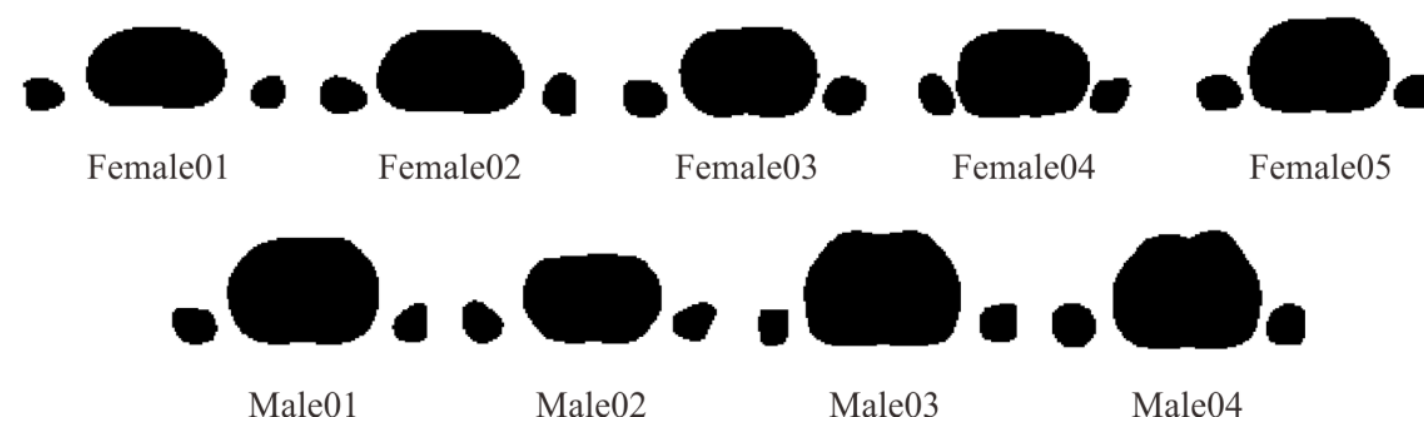

Fig. 1. The cross sections at the height of stomach of the nine voxelized subjects.

The above described simulation model has been used to analyze subject-specific radio channels in WBANs. Three different scenarios have been considered in this paper: the on-body radio channels i.e. when both the transmitter and receivers on the same subject, the inter-body radio channels i.e. when the transmitter is on one subject and receivers are on another, and the off-body radio channels i.e. when the transmitter is mounted on a fixed location in a room and receivers are on one subject. The simulation results and discussions for these three different cases are presented in the following section. 


\section{Numerical Simulation Results and Analysis}

\subsection{On-Body Radio Channels}

First the on-body radio channel is considered, i.e. the subjects are assumed to be in free space in simulations. The electric properties of human muscles are obtained from [28]. In all the simulations performed in this paper, the FDTD cell size is $\Delta x=\Delta y=\Delta z=4.0 \mathrm{~mm}$, and the time step is $\Delta t=\Delta x /(\sqrt{3} c)$, according to the Courant stability condition [13], where $c$ is the speed of light in the free space. A ten-cell Berenger's PML is used to truncate the simulation domain in all directions [13]. A patch antenna designed to operate at the wireless local area network (WLAN) frequency of $2.4 \mathrm{GHz}$ is used as the transmitting antenna (basestation) in FDTD simulations [29]. The antenna is first simulated using CST Microwave Studio ${ }^{\mathrm{TM}}$, then the radiation patterns (fields) are extracted and converted to surface currents used as excitations in FDTD simulations. The antenna is mounted on the left waist of the subjects. The whole simulation domain is divided into four, five, and five sub-domains along $x$-, $y$-, and $z$-directions, respectively. Each simulation lasts around 1.5 hours.

The path loss is calculated as the ratio of transmitted power to the received power when the steady state is reached in simulations. The receiver locations are taken on the body surface at the front and back of the subject. The receivers at the front of the body are in the line-of-sight (LOS) situation and the receivers are the back are in non-LOS (NLOS). Therefore the path loss are calculated separately for the LOS and NLOS cases and plotted in Fig. 2.
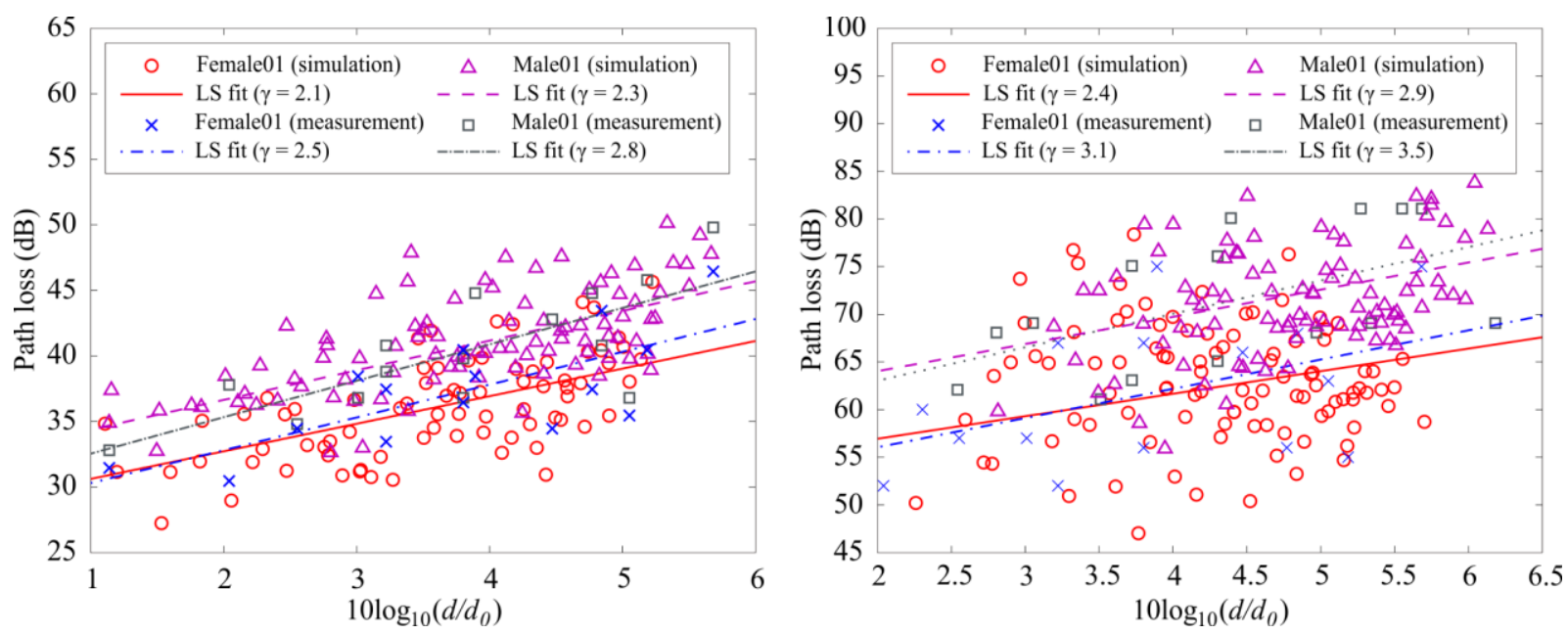

Fig. 2. The comparison of path loss (against distance) for subjects Female01 and Male01 in on-body radio channels. Left: receivers are on the front of body (LOS case), and Right: receivers are on the back of body (NLOS case).

To verify the simulation results, a site measurement is also performed in the anechoic chamber. The simulated patch antenna has been fabricated and mounted on the left waist of the subject during measurement. A printed monopole antenna is used to pick up the received power. A vector network analyzer (VNA) HP 8720 is used to connect both antennas and measure the channel response (path loss) at different receiver locations. Then a least square (LS) fitting method is used for the simulation and measurement data to obtain the path loss exponent $\gamma$ using

$$
P L(d)=P L\left(d_{0}\right)+10 \gamma \log _{10}\left(d / d_{0}\right)+X_{\sigma}
$$

where $P L\left(d_{0}\right)$ is the reference path loss at distance $d_{0}$ (taken as $d_{0}=0.1 \mathrm{~m}$ in this paper), $\gamma$ is the path loss exponent, and $X_{\sigma}$ is a zero-mean Gaussian random variable.

In general the comparison in Fig. 2 shows good agreement which validates the numerical simulation. However, the measured data is more spread comparing with the simulated ones, and this may be due to the wave reflections from cables which are not taken into account in numerical simulations. Despite this fact, the path loss exponent from simulations and measurements shows good 
agreement. To analyze the subject-specific on-body radio channels, all the nine subjects are simulated and their calculated path loss exponents are listed in Table 2.

Table 2. The comparison of path loss exponent for the nine subjects for LOS and NLOS cases in onbody radio channels ( $\mathrm{F}$ - Female, $\mathrm{M}$ - Male).

\begin{tabular}{c|ccccccccc}
\hline & F01 & F02 & F03 & F04 & F05 & M01 & M02 & M03 & M04 \\
\hline$\gamma$ (LOS) & 2.1 & 2.2 & 2.1 & 2.5 & 2.7 & 2.3 & 2.4 & 2.9 & 2.8 \\
$\gamma$ (NLOS) & 2.4 & 2.5 & 2.4 & 2.6 & 3.0 & 2.9 & 2.5 & 3.3 & 3.4 \\
\hline
\end{tabular}

By comparing with the dimensions of the subjects in Table 1, it can be seen that in general, the path loss exponent is subject-specific: the larger the size of the trunk, the higher value the path loss exponent has. The path loss exponent is larger when the receivers are on the back of the subjects, which is due to that the received signal only contains creeping waves around the body; and scattering from the arms and head occurs when the receivers are on the front of the subjects.

\subsection{Inter-Body Radio Channels}

It is also important to analyze the effect of different body sizes and shapes on the inter-body communication channels. The scenario of inter-body radio channels is illustrated in Fig. 3, with $0.04 \mathrm{~m}$ thick walls, floor and ceiling surrounding the objects added to simulate the indoor environment.

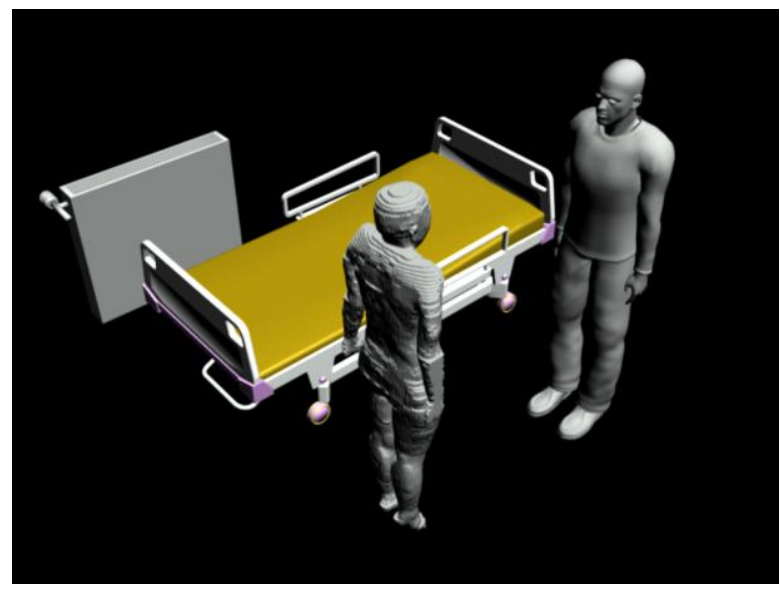

Fig. 3. The schematic diagram for the analysis of inter-body radio channels in FDTD simulations.

The size of the simulation domain is $2.2 \times 1.6 \times 1.9 \mathrm{~m}^{3}$. The transmitter is mounted on the right waist of the model on the right. The electrical properties of different materials at $2.4 \mathrm{GHz}$ used in simulations are listed in Table 3.

Table 3. Dielectric constant and conductivity of different materials at $2.4 \mathrm{GHz}$ used in parallel FDTD simulations.

\begin{tabular}{c|cccc}
\hline & Human muscle & Bed cushion & Bed frame/radiator & Walls/floor/ceiling \\
\hline$\varepsilon_{r}$ & 52.79 & 1.3 & 1.0 & 2.4 \\
$\sigma$ & 1.7 & 0.01 & $10^{6}$ & 0.15 \\
\hline
\end{tabular}

In order to investigate the impact of different subjects, the simulations are repeated by placing the receivers on all nine different models. Since for the subjects the transmitter is off-body (with respect to the left model), it is more appropriate to calculate the average values of path loss. The path loss is sampled at over one hundred locations on both the front (LOS) and back of the torso (NLOS), respectively. The path loss at different receiver locations for LOS and NLOS cases, can be well 
represented by normal distributions as shown in Fig. 4. The simulated results for the two models Female01 and Male01 are compared with measurements with good agreement.
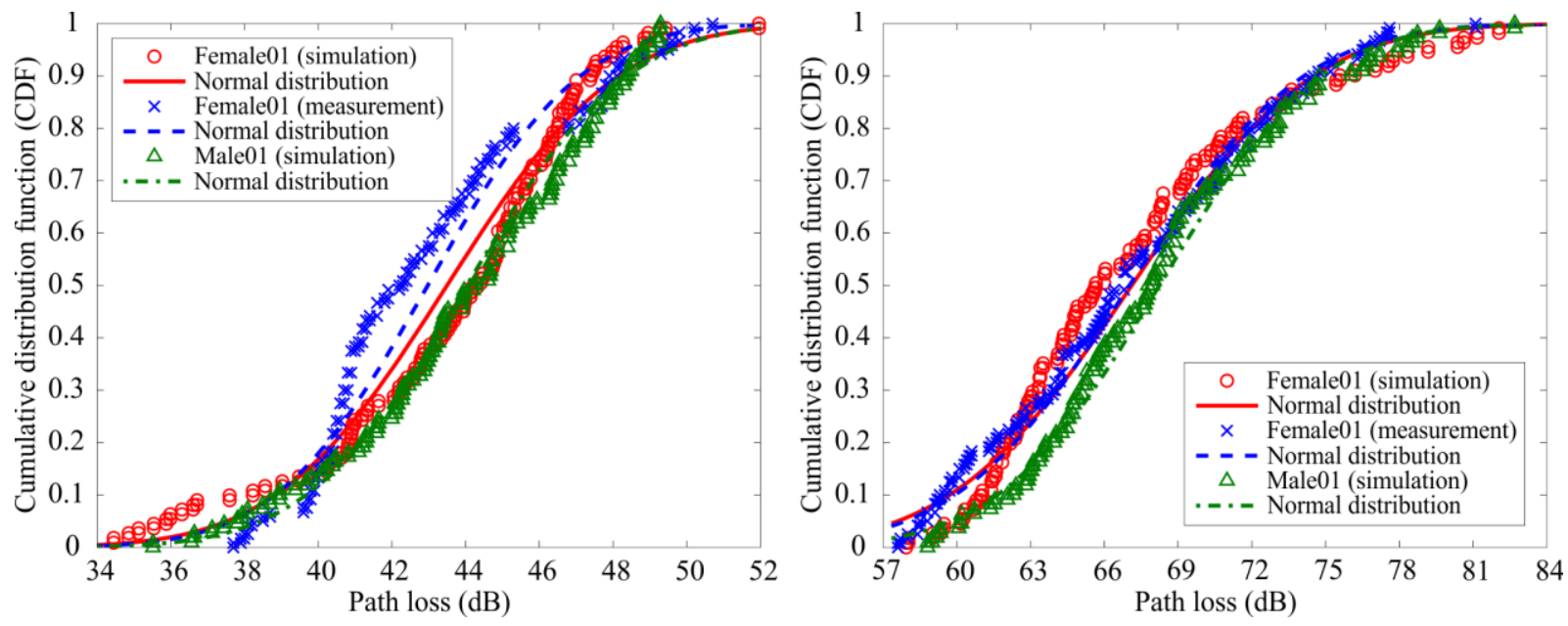

Fig. 4. The comparison of path loss (CDF) for subjects Female01 and Male01 in inter-body radio channels. Left: receivers are on the front of body (LOS case), and Right: receivers are on the back of body (NLOS case).

Figure 5 shows the mean values and standard deviations obtained for the nine different models. When the receivers are on the front of the torso (the LOS case), size and shape of the subject do not affect the propagation path, and hence the mean values of path loss are almost the same for every model. For the NLOS case, since the wave reaches the receiver mainly through reflections from the surrounding environment, the received power is not much affected by the body size, and the difference between maximum and minimum values of the average path loss (obtained for the two models Female 01 and Female04 respectively) is $4 \mathrm{~dB}$. In conclusion, since direct propagations and reflections from surrounding scatterers are the principal mechanisms of propagation, the inter-body radio channels are not subject-specific.
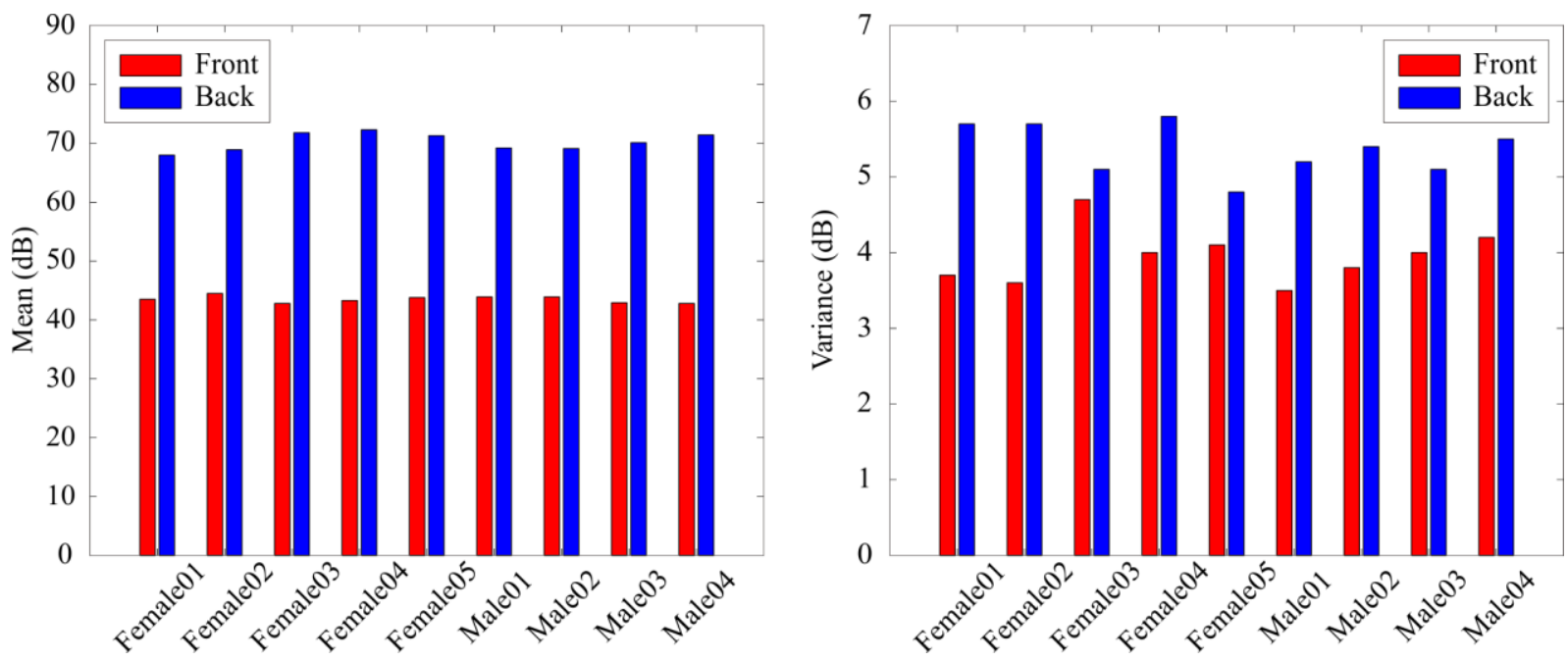

Fig. 5. The comparison of mean (left) and variance (right) of path loss values for the nine subjects for receivers on the front (LOS) and back (NLOS) of body in inter-body radio channels.

\subsection{Off-Body Radio Channels}

For the off-body radio channels, the simulated environment is similar to the one shown in Fig. 3, with the only difference is that only the subject model (the one on the left) is considered in the analysis. The 
transmitting source is placed in proximity of a wall at the height of $2.5 \mathrm{~m}$. As it was done for the interbody channels, the receivers are placed on both the front (LOS) and back of the torso (NLOS), and the communication distance is approximately $2.5 \mathrm{~m}$.

Figure 6 compares the mean values and standard deviations of path loss values for the nine subjects.
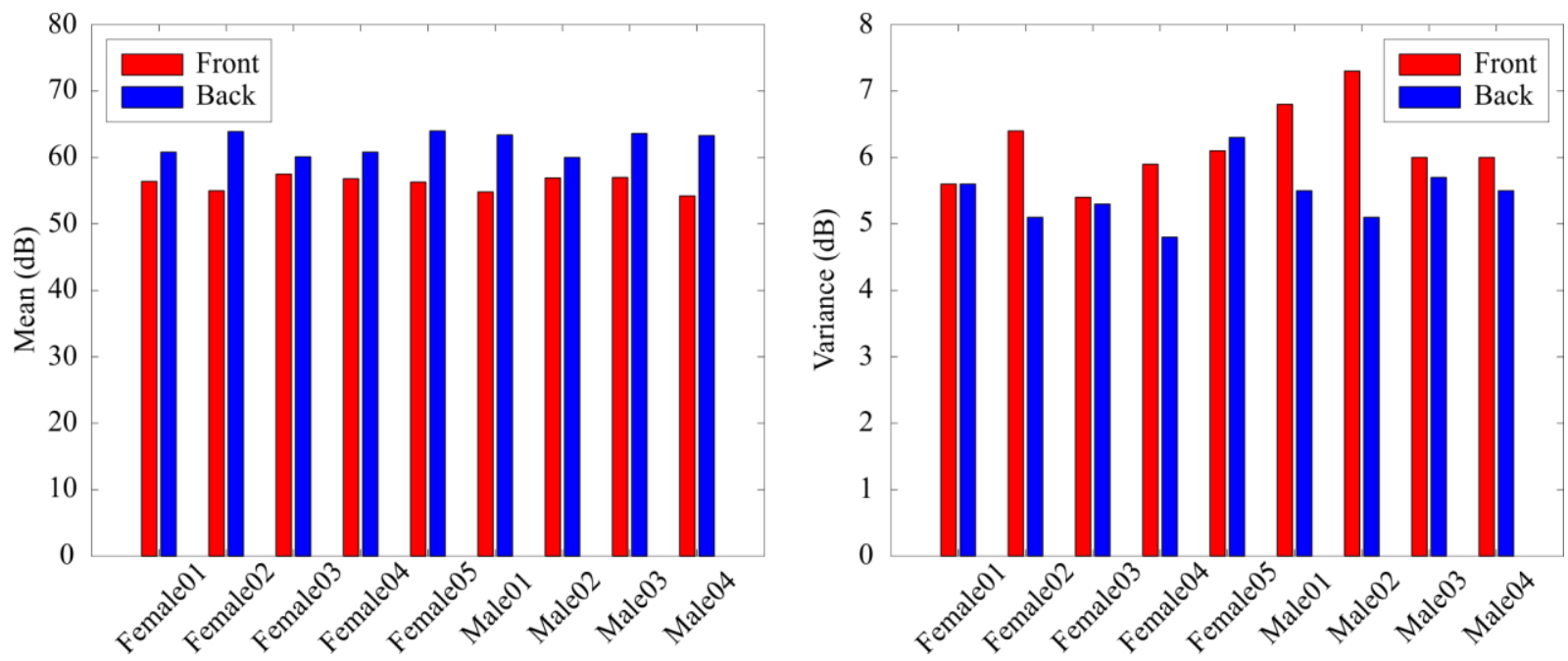

Fig. 6. The comparison of mean (left) and variance (right) of path loss values for the nine subjects for receivers on the front (LOS) and back (NLOS) of body in off-body radio channels.

The conclusions are similar to the ones drawn for the inter-body radio channel case. The differences between the maximum and minimum values of the average path loss for the LOS and NLOS cases are $3.2 \mathrm{~dB}$ and $3.9 \mathrm{~dB}$, respectively. Furthermore, the values of standard deviation do not vary much, and hence it can be concluded that the off-body radio channels are not subject-specific.

\section{Conclusions}

In this paper we present a numerical investigation of the subject-specificity of the on-body, inter-body, and off-body radio channels. The analysis of the radio channel is conducted by importing realisticshaped digital phantoms, and the simulation model has been used to study common scenario in hospital environment at $2.4 \mathrm{GHz}$. The simulation results are validated by site measurement and show good agreement. The subject-specificity of the on-body radio channel is investigated by considering nine body models of different sizes and genders, and it is concluded that parameters such as the height of the subject, and the curvature radius at the trunk affect the path loss in on-body radio channels. However, in the inter-body and off-body cases, the signal propagates away from the body and reaches the receiver through free space propagation and reflections from surrounding scatterers, and hence the impact of the subject on the radio link is found to be minimal.

\section{References}

[1] S. Drude, "Requirements and application scenarios for body area networks," Mobile and Wireless Communications Summit, IST, Jul. 2007.

[2] J. Bernardhard, P. Nagel, J. Hupp, W. Strauss, and T. Von Der Grun, "BAN-body area network for wearable computing," The 9th Wireless World Research Forum Meeting, Zurich, Jul 2003.

[3] E. Jovanov, A. O’Donnell-Lords, D. Raskovic, P. Cox, R. Adhami, and F. Andrasik, "Stress monitoring using a distributed wireless intelligent sensor system," IEEE Engineering Medicine and Biology Magazine, vol. 22, pp. 49-55, May 2003.

[4] C. Kunze, U. Grossman, W. Stork and K. Muller-Glazer, "Applications and ubiquitous computing in personal health monitoring systems," Biomedizinische Technik: 36th Annual Meeting of the German Society for Biomeidcal Engineering, pp. 360-362, 2002. 
[5] N. F. Timmons and W. G. Scanlon, "Analysis of the performance of IEEE 802.15.4 for medical sensor body area networking," $I^{\text {st }}$ annual IEEE Communications Conference on Sensor and Ad Hoc Communications and Networks (SECON), pp. 16-24, Oct. 4-7, 2004.

[6] Y. Nechayev, P. Hall, C. C. Constantinou, Y. Hao, A. Owadally and C. G. Parini, "Path loss measurements of on-body propagation channels," Proceedings of 2004 International Symposium on Antennas and Propagation, Sendai, Japan, pp. 745- 748, Aug. 2004.

[7] P. S. Hall, Y. Nechayev, Y. Hao, A. Alomainy, M. R. Kamarudin, C. C. Constantinou, R. Dubrovka and C. G. Parini, "radio channel characterisation and antennas for on-body communications," Proceedings of Loughborough Antennas and Propagation Conference, Loughborough, UK, pp. 330-333, Apr. 2005.

[8] Y. Hao, A. Alomainy, P. S. Hall, Y. I. Nechayev, C. G. Parini, and C. C. Constantinou, "antennas and propagation for body centric wireless communications," IEEE/ACES International Conference on Wireless Communications and Applied Computational Electromagnetics, Honolulu, Hawaii, USA, 3-7 Apr. 2005.

[9] A. Alomainy, Y. Hao, A. Owadally, C. G. Parini, Y. I. Nechayev, C. C. Constantinou, and P. S. Hall, "statistical analysis and performance evaluation for on-body radio propagation with microstrip patch antennas," IEEE/ACES International Conference on Wireless Communications and Applied Computational Electromagnetics, Honolulu, Hawaii, USA, 3-7 Apr. 2005.

[10] A. Alomainy, Y. Hao, C. G. Parini and P. S. Hall, "Comparison between two different antennas for UWB on-body propagation measurements," IEEE Antennas and Wireless Propagation Letters, vol. 4, pp. 31-34, Dec. 2005.

[11] A. Alomainy, Y. Hao, X. Hu, C. G. Parini and P. S. Hall, "UWB on-body radio propagation and system modelling for wireless body-centric networks," IEE Proceedings Communications-Special Issue on Ultra Wideband Systems, Technologies and Applications, vol. 153, Feb. 2006.

[12] A. Fort, C. Desset, J. Ryckaert, P. De Doncker, L. Van Biesen, and P. Wambacq, "Characterization of the ultra wideband body area propagation channel," International Conference on Ultra-WideBand, ICU'05, Zurich, Switzerland, Sep. 2005.

[13] A. Taflove, Computational Electrodynamics: The Finite-Difference Time-Domain Method, 2nd ed., Norwood, MA: Artech House, 2000.

[14] W. G. Scanlon, J. B. Burns, and N. E. Evans, "Radiowave propagation from a tissue-implanted source at $418 \mathrm{MHz}$ and 916.5 MHz," IEEE Transactions on Biomedical Engineering, vol. 47, pp. 527-534, April 2000.

[15] L. C. Chirwa, P. A. Hammond, S. Roy, and D. R. S. Cumming, "Electromagnetic radiation from ingested sources in the human intestine between $150 \mathrm{MHz}$ and $1.2 \mathrm{GHz}$," IEEE Transactions on Biomedical Engineering, vol. 50, pp. 484-492, April 2003.

[16] K. Fujii, M. Takahashi, and K. Ito, "Electric field distributions of wearable devices using the human body as a transmission channel," IEEE Transactions on Antenna and Propagation, vol. 55, July 2007.

[17] P. Salonen, K. Jaehoon, and Y. Ramaat-Samii, "Dual band E-shaped patch wearable textile antenna," IEEE Antenna and Propagation International Symposium, 2005.

[18] W. Scanlon, and N. E. Evans, "Numerical analysis of bodyworn UHF antenna systems," Electronics and Communication Engineering Journal, April 2001.

[19] M. Gallo, P. S. Hall, Y. I. Nechayev, and M. Bozzetti, "Use of animation software in simulation of on-body communication channel at $2.45 \mathrm{GHz}$," IEEE Antenna and Wireless Propagation Letter, in press.

[20] A. Alomainy, Y. Hao, and F. Pasveer, "Numerical and experimental evaluation of a compact sensor antenna for healthcare devices," IEEE Transactions on Biomedical Circuit and Systems, vol. 1, pp. 242-250, Dec. 2007.

[21] http://wwwunix.mcs.anl.gov/mpi/mpich/

[22] Y. Zhao, Y. Hao, A. Alomainy, C.G. Parini, "UWB on-body radio channel modeling using ray theory and subband FDTD method," IEEE Transaction on Microwave Theory and Techniques, vol. 54, pp. 1827-1835, Apr. 2006.

[23] L. Roelens, S. Van den Bulcke, W. Joseph, G. Vermeeren and L. Martens, "Path loss model for wireless narrowband communication above flat phantom," IET Electronics Letters, vol. 42, Jan. 2006. 
[24] E. Reusens, W. Joseph, G. Vermeeren, L. Martens, "On-body measurements and characterization of wireless communication channel for arm and torso of human," $4^{\text {th }}$ International Workshop on Wearable and Implantable Body Sensor Networks (BSN 2007), Aachen, Germany, pp. 264-269, 2007.

[25] M. J. Ackerman, "Viewpoint: The visible human project," Journal of Biocommunication, vol. 18, pp. 14, 1991.

[26] T. Nagaoka, S. Watanabe, K. Sakurai, E. Kunieda, S. Watanabe, M. Taki, and Y. Yamanaka, "Development of realistic high-resolution whole-body Voxel models of Japanese adult males and females of average height and weight, and application of models to radio-frequency electromagnetic-field dosimetry," Physics in Medicine and Biology, vol. 49, pp. 1-15, 2004.

[27] http://www.cs.princeton.edu/\$sim\$min/binvox/

[28] "An internet resource for the calculation of the dielectric properties of body tissues," Institute for Applied Physics, Italian National Research Council, http://niremf.ifac.cnr.it/tissprop/.

[29] A. Sani, Y. Zhao, Y. Hao, "Analysis of on-body antenna radiation pattern using a numerical technique based on equivalence principle and FDTD," Loughborough Antenna and Propagation Conference (LAPC '08), Loughborough, 17-18 March 2008. 
